\title{
FIGURAS DE LA CRÍTICA EN WALTER BENJAMIN: DEL ROMANTICISMO ALEMÁN A CHARLES BAUDELAIRE
}

Figures of critique in Walter Benjamin: from german romanticism to Charles Baudelaire.

Luciana Espinosa*

Resumen

En el presente artículo buscaremos analizar los diversos tratamientos que Walter Benjamin ha realizado a lo largo de su obra acerca de la crítica como temática eminentemente filosófica. Para ello abordaremos, en primer lugar, su acercamiento a la poesía del Romanticismo alemán, luego a la poesía barroca y, finalmente, a la obra de Baudelaire con el objetivo de justificar que una comprensión acabada de la crítica implica necesariamente para el filósofo alemán recuperar la actualización como clave de lectura de la historia. En efecto, si actualizar presupone para Benjamin la asunción de la no coincidencia del tiempo consigo mismo, esto es, la supervivencia del pasado en el presente y el potencial significante de sus emergencias, buscaremos mostrar cómo la noción de crítica benjaminiana descansa en ese diferencial temporal y encuentra allí su fuente de sentido.

Palabras clave: poesía, conocimiento, crítica, actualización.

Abstract

In this article we intend to analyze the different considerations that Walter Benjamin carried out in his work on the problem of critique as an eminently philosophical subject. To do this, we will address, first, his approach to German romantic poetry, then baroque poetry and, finally, Baudelaire's work, in order to explain how a full comprehension of critique means, for the German philosopher, to recover the idea of "actualization" as a key for reading History. In fact, if the actualization presupposes, for Benjamin, the assumption of the non-coincidence of time with itself, that is, the survival of the past within the present and its potentiality to signify, we try to show how Benjamin's notion of criticism rests on that temporal difference and finds there its source of meaning.

Key words: poetry, knowledge, critique, actualization.

El interés de Benjamin por la crítica es una cuestión constante a lo largo de su obra. Una de sus primeras referencias la encontramos en 1915, cuando en "La vida de los estudiantes" se compromete con una tarea liberadora de la crítica al afirmar que "solo por medio del conocimiento podemos liberar a lo futuro de su forma desfigurada en el presente. Y a esto sirve la crítica tan solo" (Benjamin, 2007, p. 78).

Unos años más tarde, entre 1917 y 1918, la problemática reaparece en el ensayo "Sobre el programa de la filosofía futura". Allí Benjamin reconoce, como corolario de su lectura entusiasta de la filosofía trascendental kantiana, que la filosofía venidera deberá necesariamente abocarse a "la tarea crítica de indagar nuevamente la posibilidad de la metafísica” (Benjamin, 1991, p. 77). 
De forma paralela, entre 1918 y 1919, el filósofo berlinés se focalizará en su proyecto doctoral cuyo resultado fue, en junio de este último año, la presentación de su tesis El concepto de "crítica de arte" en el Romanticismo alemán. En ese escrito, como su título anticipa, la crítica -en particular, la crítica de arte- se vuelve objeto de un análisis detallado a partir de su interpretación de los poetas románticos y su metafísica de la obra. Algunos años después, en 1925, Benjamin revisitará la poética barroca en El origen del drama barroco alemán, su frustrada tesis de habilitación docente. Allí justificará, por medio de la noción de crítica y su relación con la alegoría, que es posible acceder a la verdad de las creaciones tanto artísticas como filosóficas.

Se hace evidente, entonces, la continuidad del motivo de la crítica en el pensamiento benjaminiano hasta fines de los años 20, tanto como el hecho de que es recién con sus reflexiones más sistemáticas (sobre el Romanticismo y el Barroco) que este adquiere una densidad filosófica de la que carecía, producto de articularse con el trasfondo teológico-metafísico que, en ambos casos, la determina.

Pero lo mismo sucederá la década siguiente cuando Benjamin desarrolle su estudio acerca de la poesía de Charles Baudelaire. Sin embargo, en este caso, vislumbramos una leve pero decisiva modificación, ya que la problemática de la crítica si bien permanece va a resignificarse a la luz de la influencia que tanto el Surrealismo como el marxismo han tenido en su pensamiento, lo que redundará en el establecimiento de una noción distinta de la crítica, esto es, una crítica materialista.

Ahora bien, ¿por qué Benjamin recurre a la estética para desplegar sus reflexiones respecto de la crítica? ¿Por qué hacerlo, además, recuperando tradiciones poéticas casi abandonadas (Romanticismo alemán), olvidadas por la tradición (el Barroco alemán) o repudiadas por sus propios contemporáneos (Baudelaire)? En definitiva, ¿qué vínculo, si existe alguno, presupone Benjamin entre crítica e historia (del arte) para justificar su aproximación?

En las páginas que siguen nos dedicaremos a analizar el singular funcionamiento que la crítica desarrolla para Benjamin en cada uno de los horizontes artísticos mencionados: el Romanticismo, el Barroco y la modernidad de Baudelaire. No obstante, nuestro objetivo final no consiste en exponer las peculiaridades de este concepto en cada uno de los mencionados contextos históricos sino que, por el contrario, nos proponemos justificar que esas lecturas son, en sí mismas, un gesto crítico en la medida en que exponen la manera en la que el propio pensador berlinés entiende la tarea productiva de la crítica, a saber, hacer saltar la (falsa) continuidad del tiempo, evidenciar la inactualidad constitutiva del presente y producir, en el desfasaje mismo de esas temporalidades, la emergencia de nuevos significados para el ahora.

\section{LA CRÍTICA INMANENTE A PARTIR DEL ROMANTICISMO}

En 1918 Benjamin entrega en la Universidad de Berna su tesis doctoral El concepto de "crítica de arte" en el Romanticismo alemán. En ella el filósofo berlinés se propuso "presentar los fundamentos filosóficos de la crítica de arte romántica" 
(Benjamin, 1978, p. 188). Y para ello abordó problemáticamente algunos de los escritos de sus máximos representantes, en particular, de Friedrich Schlegel y Novalis (Friedrich von Hardenberg), tratando de evidenciar su relación con la herencia fichteana. En efecto, Benjamin consideraba que allí se encontraban las razones filosóficas que permitirían justificar la esencial vinculación romántica entre los motivos estéticos, gnoseológicos y metafísicos que vuelven comprensible la funcionalidad estratégica de su concepto de crítica. En palabras del propio Benjamin: "una definición del concepto de crítica de arte no se podrá pensar sin premisas epistemológicas como tampoco sin premisas estéticas; no solo porque las últimas implican a las primeras, sino ante todo porque la crítica contiene un momento cognoscitivo" (2006, p. 14).

Ahora bien, este singular vínculo que existe para los románticos entre crítica y conocimiento será el núcleo que articule la indagación benjaminiana acerca del concepto de crítica de arte. No obstante, para abordarlo habremos de rastrear, más puntualmente, la presencia de un motivo kantiano. Pues, así como la filosofía de I. Kant buscaba justificar el dominio legítimo del conocimiento humano y para ello llevó a cabo una filosofía crítica capaz de reconocer y superar los acercamientos parciales que ofrecían, por un lado, el dogmatismo (el racionalismo cartesiano) y, por otro, el nihilismo (el empirismo humeano); en el caso del Romanticismo alemán, F. Schlegel y Novalis ejecutaron la intervención equivalente pero en el ámbito estético, es decir, buscaron conciliar y superar tanto el objetivismo neoclásico como el puro subjetivismo del Sturm und Drang y lo hicieron, justamente, por medio de una reformulación del concepto de crítica (ver Abadi, 2014, p. 119ss). Efectivamente, si el dogmatismo estético encarnado en el racionalismo propugnaba un acercamiento a las obras desde la universalidad de la norma como criterio objetivo y exterior para valorarlas críticamente (el clasicismo de herencia aristotélica), en el caso del Sturm und Drang es la pura expresión de la interioridad del artista, su íntima subjetividad, la instancia que dictamina el parámetro crítico para evaluar el fenómeno artístico (las estéticas centradas en la figura del genio). De manera que la potencia del concepto romántico de crítica de arte se fundamenta en el hecho de ser una 'crítica inmanente'.

$\mathrm{Ni}$ interior ni exterior, ni objetiva ni subjetiva, la esencial determinación de la crítica de arte romántica consiste en que es la propia obra de arte particular, determinada y concreta, la que, en cada caso, es portadora de su crítica. Benjamin (2006) clarifica esta condición a partir de tres principios sintéticos: "el carácter inmediato de la evaluación, la imposibilidad de una escala de valores positiva y la no criticabilidad de lo malo" (p. 78,79).

En primer lugar, esta versión de la crítica de arte rompe con una aproximación vulgar a las obras, debido a que ella no consiste en una evaluación de las creaciones por medio de criterios externos o valores positivos (estéticos, didácticos, pedagógicos, etc.) -lo que habitualmente se conoce como "juicio de arte" y que aquí Benjamin opone a "crítica de arte" (p. 80)-. Por el contrario, la crítica inmanente es inmediata, 


\section{Luciana Espinosa}

es decir, se da junto con la obra, es coextensiva a ella. Toda obra que se precie de tal deberá poder cargar con su propia crítica (o no será verdaderamente una obra de arte).

Pero entonces, ¿en qué consiste la crítica romántica? Lo comenta Benjamin (2006), explicitando la segunda propiedad: la crítica inmanente - o crítica poética, como también se la denomina- comporta una tarea productiva y en ello radica su dimensión poética (la poética pensada como declinación de la poiesis). En este caso, la tarea de la crítica exige "exponer la relación de la obra con la idea de arte" (p. 91). Y es a esto mismo a lo que alude Benjamin cuando afirma que la crítica es semejante a un experimento, a "un experimento en la propia obra en virtud del cual se estimula la reflexión por la que la obra es elevada a la conciencia y el conocimiento de sí misma" (p. 65). Si la obra permite articular en lo sensible la idea, estaremos ante una verdadera obra de arte, ante una obra "criticable" y, en este sentido, se comprende que la crítica "lleva a priori su necesidad de existir" (p. 76). Sin embargo, si el material no es "criticable", esto es, si la "obra" no se autocoloca como instancia mediadora respecto de la idea, no estamos ante una obra sino ante una mera apariencia. Con lo que no se trataría de determinar qué obras son buenas y cuáles malas sino, más bien, de diferenciar entre obras artísticas y creaciones que no lo son. De allí, el tercer principio que enunciaba Benjamin comentando a los románticos de "la no criticabilidad de lo malo", el que puede leerse como el reverso negativo del carácter necesario de la crítica, de una crítica que define a toda obra legítima.

Resta explicitar todavía el aspecto cognoscitivo que, como mencionamos al comienzo de esta sección, estaba implicado en la formulación romántica de la crítica. Para ello debemos comprender el trasfondo metafísico de las obras en que la crítica se sustenta. Aquí la referencia es J. Fichte.

Benjamin dedica toda la primera parte de su estudio a la teoría fichteana del conocimiento y la centralidad de su concepto de Reflexión ${ }^{1}$. De hecho, la filosofía romántica reconoce allí su punto de partida, en el pensamiento inmediato que se refleja sobre sí mismo como autoconciencia, esto es, la Reflexión. Esta primera instancia del ser, absoluta, será la que posibilite, por medio de ciertas mediaciones sucesivas, las demás entidades y relaciones, cada vez más mediatas. En este sentido, hay que destacar que la naturaleza reflexiva del pensamiento funciona como una garantía en relación con el carácter intuitivo e inmediato de todo conocimiento dado que en última (o primera) instancia todo remite a ese "pensarse-a-sí-mismo" como fenómeno fundamental ${ }^{2}$.

\footnotetext{
${ }^{1} \mathrm{Al}$ respecto, debido a la preponderancia que el análisis benjaminiano dedica a la Reflexión, W. Menninghaus ha sugerido que el verdadero título del trabajo debería haber sido "La teoría de la Reflexión poética en el Romanticismo alemán" (Menninghaus, 2002, p. 20-50).

${ }^{2}$ Este compromiso con la Reflexión hace estallar el planteo clásico de la gnoseología que opone sujeto y objeto. Benjamin lo enfatiza al afirmar que "todo conocimiento es una conexión inmanente en el absoluto, o si se quiere, en el sujeto. El término objeto no designa una relación en el conocimiento sino más bien una falta de relación y pierde su sentido dondequiera que se presenta una relación de conocimiento. (...) El conocimiento está anclado en la reflexión: el ser conocido de una esencia a través de otra coincide con el autoconocimiento de lo que está 
Así, esta disolución de la mediatez en el absoluto de la Reflexión permite afirmar que todo conocimiento es necesariamente inmediato, que no hay una relación cognoscitiva extrínseca sino más bien que conocer y percibir se identifican en la Reflexión. Y es esto mismo lo que sucede con las obras de arte. Para los románticos, el arte en general es un médium de la Reflexión y la obra un centro específico y puntual de ella (la Reflexión). De forma que la crítica de arte como crítica poética o crítica inmanente "no consiste en la reflexión sobre un producto (...) sino en el despliegue de la reflexión, esto es, del espíritu, en el producto" (Benjamin, 2006, p. 67).

En conclusión, la Reflexión como substrato metafísico habilita una noción de crítica que, como ya mencionamos, consiste en la exposición de la relación de una obra particular con todas las demás obras y, en definitiva, con la idea misma de arte. Así entendida, la crítica inmanente por un lado consuma y completa la obra particular, y por otro, la disuelve en el Absoluto hasta fusionarla con la idea (Mosès). De modo que como un procedimiento redentor, como instancia que según Benjamin (1990), permitirá "el despertar (...) de la conciencia en las obras vivas" (p. 175), la crítica culmina el ciclo vital de las obras pero que es, en última instancia también, el ciclo de la trama viviente de todo el universo romántico.

\section{LA ALEGORÍA BARROCA COMO FORMA DE LA CRÍTICA}

Tras la aprobación de su tesis doctoral, Benjamin se dedicó a profundizar los estudios acerca de G. W. Goethe que había comenzado a desarrollar allí, en el apartado final "La teoría del arte temprano-romántica y Goethe". Esta investigación culminó años más tarde, en 1922, con la publicación de un ensayo crítico titulado Sobre las afinidades electivas de Goethe en el que, como señala B. Witte, Benjamin "satisface de manera paradigmática las exigencias que había formulado como necesarias para la crítica en su tesis" (Witte, 1990, p. 56). Es decir, el corpus teórico que se había desarrollado hasta ese momento es puesto en práctica por Benjamin en esa "crítica ejemplar" -como él mismo caracteriza a ese ensayo-, mostrando de qué manera era posible efectuar una crítica inmanente de las obras, comprometiéndose con la autonomía de sus criterios para "iluminar[la] absolutamente a partir de sí misma" (Benjamin, 1996, p. 46).

Sin embargo, recién en 1925, cuando Benjamin redacte su tesis de habilitación docente, El origen del drama barroco alemán, encontraremos una nueva formulación de la crítica. Según consta en una carta enviada a G. Scholem en abril de ese mismo año, el objetivo del escrito partía de una "exigencia autoimpuesta" que buscaba realizar una "indagación crítica de la forma del drama barroco alemán", para la que sería preciso "desarrollar una serie de argumentaciones metodológicas sobre la crítica” (Steiner, 2014, 287).

siendo conocido, con el del cognoscente y con el ser conocido del cognoscente a través de la esencia que conoce" (Benjamin, 2006, p. 58). 


\section{Luciana Espinosa}

De forma análoga a lo que sucedía en el Romanticismo, el Barroco le ofrece a Benjamin un horizonte específico y particular a partir del cual desplegar la problemática de la obra y de la crítica (Bégot, 2013). Contemporáneo a la Reforma ${ }^{3}$, el Barroco alemán se caracteriza por la afirmación de la inmanencia, el total vaciamiento de una naturaleza que, en condición de caída ${ }^{4}$, "es sentida como eterna caducidad" (Benjamin, 1990, p. 173). Como precisa B. Lindner (2014) acerca de esta cuestión, la cosmovisión propia del drama barroco alemán reclama para su justa comprensión, "una referencia a su horizonte teológico-político, horizonte marcado por la caída de toda escatología (...) que determina la inmanencia radical y la forzada terrenalidad del Barroco" (Lindter, 2014, p. 25).

Lejos de "la apariencia luminosa de la idea" (Benjamin, 1990, p. 173) que dominaba el planteo anterior, Benjamin se enfrenta aquí con que "la falsa apariencia de totalidad se extingue (...) el eidos se apaga (...) y el cosmos contenido en ella se seca" (p. 169). De este modo, serán las ruinas y los fragmentos, "lo que yace reducido a escombros, el material más noble de la creación en el Barroco" (p. 171). Material a partir del cual emergerán las alegorías como sus obras paradigmáticas en la medida en que ellas "encuentran en la disolución de la falsa apariencia de totalidad la fuerza de su potencia significante" (p. 159). Disolución que, obviamente, implica ir más allá de una estética de lo bello (como es el caso del Romanticismo) y hace de "lo muerto" y "lo inexpresivo" sus nuevos componentes esenciales:

tal es el núcleo de la visión alegórica: a mayor significación, mayor sujeción a la muerte, pues es la muerte la que excava más profundamente la abrupta línea de demarcación entre la Physis y la significación. Pero si la naturaleza ha estado desde siempre sujeta a la muerte, entonces desde siempre ha sido también alegórica (Benjamin, 1990, p. 159).

Este íntimo vínculo que Benjamin reconoce -siguiendo a los poetas del sigo XVII alemán- entre naturaleza, muerte y significación se consuma entonces en la alegoría, es decir, encuentra en ella su materialización artística. No obstante, es fundamental resaltar que no se trata de pensar las alegorías barrocas como un mero procedimiento estético ni como un tropo determinado de la historia del arte o una "técnica gratuita de producción de imágenes" (Benajmin, 1990, p. 155); por el contrario, las alegorías son para Benjamin "expresión, del mismo modo que el lenguaje

\footnotetext{
${ }^{3}$ Respecto de este punto, Benjamin afirma en el libro acerca del Barroco: "En aquella reacción excesiva que, a fin de cuentas, excluía las buenas obras en cuanto tales, y no solo por su carácter de mérito y de expiación, se manifestaba un componente de paganismo germánico y de oscura creencia la sujeción al destino. Algo nuevo surgió: un mundo vacio" (El origen, 131, énfasis mío).

${ }^{4}$ Benjamin en 1916 redactó tres artículos en los que despliega su propia teoría del lenguaje: "El significado del lenguaje en el Trauerspiel y en la tragedia", "Trauerspiel y Tragedia" y "Sobre el lenguaje en general y sobre el lenguaje de los hombres" en los que se plantea la singular relación que él estipula entre lenguaje, naturaleza y la relación con la Creación. Para una interpretación que hace especial hincapié en el carácter caído de la naturaleza en términos lingüísticos (ver Espinosa, 2012).
} 
o la escritura" (p. 155). Esta afirmación, de apariencia sencilla, debe ser puesta en conexión con los trabajos tempranos del pensador alemán, en que justifica, por medio de su teoría del lenguaje y la traducción (ver Benjamin, 1967), que el ser de los nombres (el ser que constituye el lenguaje y la escritura) es el ser al que está orientada la tarea filosófica ${ }^{5}$. De modo que al comprometerse con las alegorías como expresión, Benjamin les otorga el más alto reconocimiento y les asigna un propósito filosófico de primer orden.

Ahora bien, ¿qué es lo que ellas expresan? La respuesta la ofrece Benjamin sirviéndose de una contraposición con el símbolo romántico:

mientras en el símbolo, con la idealización de la destrucción, el rostro transfigurado de la naturaleza se muestra fugazmente a la luz de la redención, en la alegoría la facies hipocrática de la historia yace ante los ojos del observador como paisaje primordial petrificado. Todo lo que la historia desde el principio tiene de intempestivo, de doloroso, de fallido, se plasma en un rostro; o mejor dicho: en una calavera (Benjamin, 1967, 159).

Las alegorías, las obras barrocas por excelencia, son las instancias que logran exhibir la constitución sufriente de su propio tiempo, el desgarramiento de una naturaleza mortificada que vivencia el alejamiento de la divinidad, esto es, su propia orfandad como condición insuperable. Y es eso mismo lo que ellas expresan: la imposible clausura de una realidad desolada que significa siempre y solo su muerte. Por tanto, expresar no implica representar, reunificar o reconciliar sino "hacer justicia" al darse de un mundo quebrado.

Pero la alegoría como expresión es fundamental, además, en otro sentido y es que como afirma Steiner comentan el texto benjaminiano, ella "anticipa el proceder de la crítica" (p. 288). Pues esta última, en el trabajo acerca del drama barroco alemán es presentada como "mortificación de las obras" (Benjamin, 1990, p. 175). Y si asumíamos que las alegorías encuentran en la muerte y la fragmentación de la totalidad su propia configuración, ellas serán la precondición para el advenimiento de la crítica en la medida en que esta podrá revelar, sin miramientos, la íntima constitución de las creaciones. Es decir, la crítica una vez más, es la manera en que Benjamin plantea el acceso cognoscitivo de las obras. Es importante, sin embargo, aclarar que, a diferencia del Romanticismo, el conocimiento no implica para el siglo XVII un saber respecto de

\footnotetext{
${ }^{5}$ En el prefacio del libro respecto del barroco alemán "Algunas cuestiones preliminares de crítica del conocimiento", Benjamin ejecuta un particular guiño a Platón y a Leibniz al afirmar que la tarea del filósofo consiste en ejercitarse en trazar una descripción del mundo de las ideas (a las que compara, explícitamente, con las mónadas). Entendidas como constelaciones eternas que reúnen la multiplicidad de lo real sin reducción alguna, las ideas tienen a cargo la tarea de salvar los fenómenos por ella reunidos, pues, a diferencia del concepto que propone una síntesis de lo diverso y se propone una lógica explicativa en términos deductivos, ella desde su lógica expresiva únicamente los afirma y los reúne. Con ello, hay sin dudas una relación íntima entre filosofia e ideas para Benjamin pero también entre ideas y expresión, ya que su ser es lingüístico (ver Benjamin, 1967, p. 15-22)
} 


\section{Luciana Espinosa}

instancias positivas sino que, en tanto nos referimos a las alegorías, se trata de un saber de algo ya perimido: "no se trata, a la manera romántica, de un despertar de la conciencia en los vivos, sino de un asentimiento del saber en las obras que están muertas" (p. 175).

Con esto, la crítica como mortificación de las obras implica para Benjamin la posibilidad de su conocimiento y, por tanto también, la exposición en su verdad. Verdad que abandona en el mundo barroco su declinación tradicional -como adecuación entre el plano del decir y del ser-para ser entendida como "la muerte de la intención"(p. 18).

Otra vez el motivo de la muerte sale al encuentro pero en su insistencia nos permite hilvanar las temáticas de la alegoría, la crítica, el conocimiento y la verdad como una sola cuestión compleja. En el esquema de la significación alegórica, la expresión que ellas son expone su verdad, verdad que "no es un desvelamiento que anula el secreto sino una revelación que le hace justicia" (Benjamin, 1990, p. 13); en otras palabras, la alegoría se da como forma de la crítica y la crítica permite conocer la verdad de las obras alegóricas. De manera que Benjamin, en su acercamiento a la poesía barroca, abordó la cuestión de la crítica de manera análoga a la que había ejecutado en su lectura del Romanticismo, solo que con el signo contrario: no afirmando la plenitud vital del universo sino la insoslayable muerte que lo determina.

Ahora bien, ¿qué pasa con la crítica en la producción benjaminiana de la década siguiente, en los años 30 ? ¿Cómo se articula en el contexto de su reflexión pertinente al siglo XIX? A esbozar algunas posibles respuestas nos dedicaremos en el siguiente apartado.

\section{LA NOCIÓN MATERIALISTA DE LA CRÍTICA A PARTIR DE BAUDELAIRE}

Así como fueron los poetas barrocos con el siglo XVII y los románticos con el XVIII, será la poética de Charles Baudelaire la encargada de despertar las consideraciones benjaminianas acerca de la crítica en el siglo XIX. Pero a diferencia de las figuras de la crítica mencionadas en los apartados anteriores, Benjamin no retomará de los poemas baudelerianos un tratamiento o definición explícita de la crítica sino que, por su intermedio, buscará extraer su gesto crítico. De hecho, en una carta escrita a fines de los años 30, Benjamin sostiene que en el trabajo concerniente a Baudelaire se había propuesto "convertir la poesía del siglo XIX en un médium de su conocimiento crítico" (Benjamin, 1985, p. 228). Con lo que, una vez más, la poesía se presenta como ocasión del despliegue de una reflexión referida a la relación cognoscitiva que se realiza por medio de la crítica.

En el caso de la poesía baudelairiana sobresale para Benjamin que "no solo es susceptible, como cualquier otra, de una determinación histórica, sino que quiso serlo y así es como se entendió a sí misma" (Benjamin, 1972, p. 132). Es decir, la obra de Baudelaire está tramada de forma tal que en su densidad estética aparece, deliberadamente, un índice histórico que la remite de manera inmediata a su propio tiempo y, por eso mismo expone las condiciones materiales que hicieron posible su surgimiento. 
En muchos casos estas determinaciones históricas son el motivo explícito de los versos baudelairianos, pero en otros ellas aparecen camufladas "tras los motivos del satanismo, el spleen y el erotismo desviado" (Benjamin, 2002, p. 153). Por tanto, una ciudad camino a ser obra monumental a manos de Haussmann, una metrópolis colmada de nuevos personajes que la obligan a alterar su antiguo rostro (la prostituta, el comerciante, el vagabundo, el dandy, etc.), un Paris que comienza a acelerar su pulso al ritmo vacío de las mercancías, pero sobre todo, un sitio que propone la experiencia misma de lo nuevo son algunos de los elementos de esa "impresionante fenomenología de la modernidad metropolitana" (Lindner, 2014, p. 53), que se vuelve, por primera vez, poema con Baudelaire.

Pero para el poeta del siglo XIX, la gran ciudad, la sociedad de masas y la dinámica capitalista si bien son la novedad, aquello que irrumpe inesperadamente con la modernidad, son también su más infernal reverso, el ciclo infinito de lo siempre igual que solo se quiebra con la muerte ${ }^{6}$. Lo que explica el posicionamiento fuertemente ambivalente que el poeta francés le atribuye a esta configuración sociohistórica de Paris y que será recuperado por Benjamin para pensar la crítica en este nuevo contexto.

No obstante, además de desarrollarla en sus temáticas, la determinación histórica de los escritos de Baudelaire se condensa en la singular trama alegórica que los constituye. Tout pour moi devient allégorie (Benjamin, 1972, p. 184) es el verso del poema baudelairiano Le cygne que Benjamin elige como epígrafe para la sección que en Paris, capital del siglo XIX dedicará al poeta y que se inaugura con la afirmación que reconoce que "el ingenio de Baudelaire (...) es alegórico" (p. 184).

Como en el Barroco, la alegoría es un elemento central en la poética baudelairiana. Pues, si su fuerza significante se despliega allí donde "el duelo por lo que fue y la desesperanza por lo que vendrá" (Benjamin, 1972, p. 101) es la constante, tanto el Barroco como la modernidad parisina son configuraciones epocales especialmente proclives a su fuerza expresiva. Sin embargo, más allá de las continuidades, hay en la alegoría moderna una importante diferencia respecto de su antecedente barroco y es que la alegoría baudelairiana "habla no solo de un desalojo de la totalidad, sino también de las bases sociales de ese desmoronamiento" (García, 2010, p. 170).

En efecto, si la alegoría del siglo XVII tenía como motor expresivo el alejamiento de la trascendencia y la plena afirmación de la inmanencia que aparece como su contracara, la alegoría moderna sumará a su fuerza significante el devenir dominante del "primer capitalismo avanzado" (Benjamin, 2005, p. 385) que, según consigna Baudelaire, ha implicado el extravío de las condiciones tradicionales de la experiencia sin haber estipulado las coordenadas específicas de sus expectativas futuras. Un nuevo vaciamiento tiene entonces lugar en el siglo XIX pero ahora no de la divinidad sino de la densidad de una experiencia que ha "triturado [su] aura en la vivencia del shock" como único "precio al que puede tenerse la sensación de lo

\footnotetext{
${ }^{6} \mathrm{Al}$ respecto, Benjamin (2002) afirma: "Para los hombres tal como son actualmente hay solamente una novedad radical, y es siempre la misma: la muerte" (p. 154).
} 


\section{Luciana Espinosa}

moderno" (Benjamin, 1972, p. 170). Es que, justamente, el fundamento de la intención alegórica para Baudelaire se encuentra allí, en "la devaluación específica del mundo de las cosas que se manifiesta en la mercancía” (Lindner, 2014, p. 54).

Se puede comprobar entonces que la alegoría baudelariana no es para Benjamin un mero procedimiento artístico o una herramienta metodológica aleatoria (como tampoco lo era en el Barroco), sino que es portadora del valor filosófico más alto en tanto de ella depende la posibilidad de expresar una experiencia fuertemente conmovida -en este caso, a manos de la mercancía- y, por tal razón también, de formular su crítica. Nuevamente, como sucedía en el Barroco, la profundidad de la significación alegórica hace de ella la instancia portadora de la más alta tarea crítica. De hecho, podríamos arriesgar que la alegoría es para el pensador alemán, la forma moderna de la crítica. Pero ¿en qué sentido debemos entender esto?

El acercamiento benjaminiano a Baudelaire parece así implicar una reformulación respecto de las nociones románticas y barrocas de la crítica, porque la crítica aquí será una crítica materialista. Si antes la estructura metafísica era la que imprimía el contorno específico al trabajo y la función de la crítica -ya sea recurriendo a la Idea o a la Reflexión propias del Romanticismo o a la naturaleza caída en el barroco-, aquí dicha relación se encuentra modificada.

Esta nueva condición material de la crítica implica que las alegorías en su proceder expresivo y significante, lograrán quebrar la cómoda habitualidad de una experiencia adormecida que, en el Paris del siglo XIX, se encontraba en vías de desaparecer. Y lo hacen debido a que pueden dar cuenta de las contradicciones en las que se asienta la modernidad capitalista. Pues si las alegorías "detentan la función de expresar poéticamente la experiencia urbana de Baudelaire", habremos de reconocer junto con Benjamin que esta expresión poética es también una expresión política; una instancia capaz de ejecutar la crítica de las condiciones materiales (sociales, económicas, políticas, etcétera) de su aparecer.

En este sentido, nos vemos tentados de hipotetizar una secreta afinidad entre el crítico y el historiador materialista que, durante estos mismos años, tematizara Benjamin (2012) en su exilio parisino (p. 387-406). En efecto, ambos hacen de la lectura la clave de su función: si el crítico es quien debe leer las condiciones materiales alegorizadas en las obras, el historiador materialista es quien tiene que "leer lo que nunca fue escrito" (p. 404) de la historia. En los dos casos, son los silencios y las omisiones, las oscuridades y las opacidades propias de su objeto (las obras y el pasado) las instancias más productivas y, por eso mismo también, las cargadas de mayor fuerza crítica.

\section{CONSIDERACIONES FINALES}

Como hemos intentado mostrar, Benjamin ha hecho de las distintas figuras de la crítica, es decir, de las diversas declinaciones puntuales que esta va asumiendo a lo largo de la historia del arte que él aborda, un aspecto central de su reflexión filosófica. Sin pretender dar una imagen total o una "teoría general" de la misma sino, simplemente, 
buscando aportar algunos elementos que nos permitan entrever el gesto crítico presente a lo largo de sus obras, creemos que es preciso recuperar una convicción filosófica fundamental que aquí ha estado operando sin explicitarse: la actualización como forma de la crítica.

Pues sería equivocado pensar que las investigaciones históricas llevadas adelante por Benjamin buscan, por ejemplo, analizar el carácter inmanente de la crítica romántica así como esta era en sí misma, indagar la mortificación que efectuaban las alegorías barrocas en tanto instancias críticas de su propio tiempo, o examinar las contradicciones materiales que se visibilizan por medio de las alegorías modernas como figuras perimidas de la crítica. Por el contrario, el cometido parece ser otro, mucho más relevante: mostrar por su intermedio de qué manera ellas, en el desfasaje de los tiempos implicados, permiten la emergencia de un nuevo sentido cuyo efecto se visualiza siempre en el corazón mismo del presente.

Así, la aproximación benjaminiana a la poesía romántica, barroca o baudelairiana no las aborda como fenómenos de la historia (del arte) sino más bien en lo que ellas tienen para ofrecer en un tiempo que no es el suyo, al presente de Benjamin. Y es este el sentido en que entendemos la crítica como una cuestión de actualización, pues, actualizar el pasado es para el pensador berlinés un gesto político que consiste en acercarse a él a base de una preocupación presente en donde tanto el pasado como el presente quedan, sin dudas, modificados. Con ello, si la actualización implica comprometerse con la no coincidencia del tiempo consigo mismo y la supervivencia del pasado (que espectraliza y engrosa el presente), la crítica como actualización se convierte en una tarea política.

Llegados a este punto, se comprende la real dimensión de la noción benjaminiana de crítica como un asunto netamente filosófico que se sostiene en el abismo diferencial de los tiempos. Y es que solo así, en la torsión propia del ahora y la aceptación de lo sido y lo imprevisto que lo atraviesa, se puede comprender que "la exposición de la historia lleva al pasado a colocar al presente en una situación crítica" (Benjamin, 2005, p. 473).

Universidad de Buenos Aires* Instituto de Investigaciones Gino Germani, Facultad de Ciencias Sociales Consejo Nacional de Investigaciones Cientificas y Tecnológicas (CONICET) Pte. J. E. Uriburu 950, 6to piso (1114), Ciudad Autónoma de Buenos Aires (Argentina) lu.espinosa@gmail.com

\section{OBRAS CITADAS}

Abadi, Florencia. (2014). Conocimiento y redención en la filosofia de Walter Benjamin. Buenos Aires: Miño y Dávila.

Bégot, Jacques-Olivier. (2013). 'Le théâtre du salut. L'esprit de l' allégorie et son destin dans le Trauerspiel'. Revue littéraire Europe, No 1008, año 91. Paris: CNL, 69-79. 
Benjamin, Walter. (2012). Escritos franceses. Buenos Aires: Amorrortu.

— (2007). Obras. Libro II, Vol. 1, Madrid: Abada Editores.

— (2006). "El concepto de 'crítica de arte' en el Romanticismo alemán”. Obras, Libro I, Volumen I. Madrid: Abada.

— (2005). El libro de los pasajes. Madrid: Akal.

— (2002). "Parque central" en Ensayos I. Madrid: Editora Nacional.

_ (1996). "Las afinidades electivas de Goethe". En Benjamin Walter, Dos ensayos sobre Goethe. Barcelona: Gedisa.

_ (1991). "Sobre el programa de la filosofía venidera". Para una crítica de la violencia y otros ensayos. Iluminaciones $I V$. Madrid: Taurus.

— (1990). El origen del drama barroco alemán. Madrid: Taurus.

- (1985). GS VI. Herausgegeben von Rolf Tiedemann und Hermann Schweppenhäuser, Frankfurt am Main, Suhrkamp Verlag.

_ (1978). Briefe. Hrsg. und mit Anmerkungen versehen von Gershom Scholem und Theodor W. Adorno. 2 vv. $2^{\text {a }}$ ed. Frankfurt/M: Suhrkamp.

_ (1972). "Sobre algunos temas en Baudelaire". Iluminaciones II, Madrid: Taurus.

— (1967). Ensayos escogidos. Buenos Aires: Editorial Sur.

Espinosa, Luciana. (2012). Naturaleza caída y sujeto melancólico: una lectura desde la filosofia del lenguaje de Walter Benjamin. Tópicos, (24), 00. Recuperado en 01 de octubre de 2017, de http://www.scielo.org.ar/scielo.php?script=sci_arttext\&pid= S1666-485X2012000200003\&lng=es\&tlng=es.

García, Luis. (2010). "Alegoría y montaje. El trabajo del fragmento en Walter Benjamin". Constelaciones. Revista de teoría crítica, Vol. 2, 158-185.

Lindner, Burkhardt. (2014). "Alegoría”. Opitz, M., Wizisla, E. (comps.). Conceptos de Walter Benjamin, Buenos Aires: Editorial Las cuarenta.

Menninghaus, Winfried. (2002). "Walter Benjamin's exposition of the romantic theory of reflection", en Hanssen, B. y Benjamin, A. (Comps.), Walter Benjamin and Romanticism. Londres-Nueva York: Continuum.

Mosès, Stephan. (2013). "Walter Benjamin et le romantisme allemand". Revue littéraire Europe. № 1008, Año 91, 57-68.

Steiner, Uwe. (2014). "Crítica" en Opitz, M., Wizisla, E. (Eds.), Conceptos de Walter Benjamin. Buenos Aires: Editorial Las Cuarenta.

Witte, Bernd. (1990). Walter Benjamin. Una biografia. Buenos Aires: Mila/AMIA. 